John Rink

\title{
Chopin Copying Chopin
}

It is tempting to think of Chopin as a creative genius whose ideas poured forth from his pen - but the act of notating his music was at best a chore if not a downright penance for

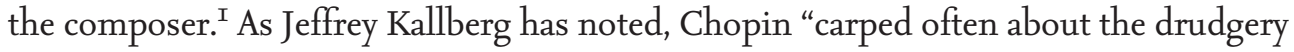
of writing out his music and liked to minimize all copying". ${ }^{2}$ The need to copy out his own scores increased in the I840s when Julian Fontana departed for America, leaving Chopin without his most reliable amanuensis. This forced him to produce several autograph Stichvorlagen for his respective publishers in Paris, London and Leipzig, an especially tedious process compared with those in use before. ${ }^{3}$

It is my contention that within these multiple autographs, prepared at more or less the same time, Chopin left valuable evidence not only of his musical creativity (to wit the numerous compositional variants found in discrete versions of many works) but of notational habits possibly formed earlier in his career when producing individual autograph Stichvorlagen on the basis of antecedent drafts. Although Chopin's musical variants have attracted considerable scholarly attention, ${ }^{4}$ much less has been devoted to the notational discrepancies between different versions ${ }^{5}$ - discrepancies which in principle could have significant implications for the music's content as realised in any given performance. The systematic study of such discrepancies is required not only to gain insight into the meaning of individual signs and symbols, the copying practices, and the revision-

1 A precursor to this essay was presented at a conference organised by the Chopin Institute in 2002 and then published in Chopin's Work. His Inspirations and Creative Process in the Light of the Sources, ed. by Artur Szklener, Warsaw 2003, pp.67-8I; see also the Polish translation: Chopin kopiujący Chopina, in: Studia Chopinowskie I (20I8), pp. 20-39. Original citations in Polish may be found there.

2 Jeffrey Kallberg: Chopin at the Boundaries. Sex, History and Musical Genre, Cambridge, MA/London I996, p. IоI.

3 For discussion of the publication history of Chopin's music, see Christophe Grabowski/John Rink: Annotated Catalogue of Chopin's First Editions, Cambridge 2010.

4 See for example Jeffrey Kallberg: The Chopin "Problem". Simultaneous Variants and Alternate Versions, in: id.: Chopin at the Boundaries, pp.215-228; and John Rink: Playing with the Chopin Sources, in: Chopin et son temps/Chopin and his Time, ed. by Vanja Hug and Thomas Steiner, Bern 20I6, pp. 4I-53.

5 Such discrepancies are discussed in individual volumes in the Wydanie Narodowe series (see for example note I4 below) and The Complete Chopin - A New Critical Edition; see also Jan Ekier: On Questions Relating to the Chronology of Chopin's Works. Methods. A Few Examples Concerning Compositions from the Last Period, in: Chopin's Musical Worlds. The I840's, ed. by Artur Szklener, Warsaw 2008, pp. I69-I88. 
ist tendencies that possibly gave rise to them, but also to develop more informed approaches to editing Chopin's music. ${ }^{6}$

The focus of this essay is therefore on notational divergences between original and later versions of Chopin's compositions, those later versions taking the form of copies explicitly prepared as such soon after the composition of the former. Matters such as layout and the disposition of pitches as well as a host of additional parameters are addressed with regard to the three autograph Stichvorlagen for the Barcarolle Op. 6o, two of which survive in an immediately accessible form. Before turning to them, however, I will identify the four types of copy that Chopin could be said to have prepared at different points in his career or at successive compositional stages. The first type concerns what I regard as "copies" internal to a work - for instance, of parallel passages, whether verbatim or transposed; the second type, copies of entire works, made at the time of the music's original composition; the third type, copies of entire works, produced at an extended interval after the music's original composition but based on earlier versions; 7 and the fourth type, copies prepared on the basis of, but departing from, earlier (rejected) versions. ${ }^{8}$ Other possibilities include multiple copies made in succession (as in the case of the work investigated here, the Barcarolle) and, lastly, some combination of the above. It will be useful to keep these different types in mind during the ensuing discussion, which obviously cannot be exhaustive in this context.

I have identified three main categories of discrepancy among Chopin's multiple copies: those reflecting errors on the composer's part; those constituting intentionally new material; and those arising from what I see as notational alternatives, on which I focus in due course. Errors might have resulted from accidental omissions, Chopin's misreadings of his own script, the wrong material being copied, and other mistakes caused by carelessness or inattention; examples include incorrectly placed pedal signs or releases, inconsistent beginnings and ends of slurs, and so on. Changes amounting to intentionally new content might encompass corrections of earlier errors such as omis-

6 See Wojciech Nowik: The Receptive-Informational Role of Chopin's Musical Autographs, in: Studies in Chopin, ed. by Dariusz Żębrowski, Warsaw I973, pp. 77-89; see also id.: Autografy muzyczny jako podstawa badań źródłowych w Chopinologii, in: Muzpka I6/2 (I97I), pp. 65-84. For more general discussion, see James Grier: The Critical Editing of Music, Cambridge I996.

7 An example of this type of copy would be the partial autograph of the Concerto in $\mathrm{f}$ minor, produced not in I830 when the piece was composed but in circa I835. For discussion, see John Rink: Chopin. The Piano Concertos, Cambridge I997; and id.: Chopin. Koncerty fortepianowe, trans. by Aleksander Laskowski, Warsaw 2015. I do not include presentation manuscripts in this category because most were not prepared as "copies".

8 Examples include the Ballade Op.52 and Mazurka Op.59 No.2. 
sions; variants of or alternatives to earlier material; and interpolations and other additions such as fingering, expressive indications, articulation, et cetera.

The preceding types of discrepancy are well known to anyone familiar with the Chopin sources, but the third category - notational alternatives - is less widely acknowledged. Such alternatives can be divided into two groups: those which appear to be equivalent in intention and effect (for example, cres - - - and cres — might seem equivalent but potentially have different musical effects (for example, $\boldsymbol{f}>$ and $\boldsymbol{f}=$ ). The latter are of particular import in this study and indeed in editorial work on Chopin more generally. For that reason, I seek conclusions which are typological in nature and allow one to define a "notational lexicon", as well as conclusions which stem from the particular logic underlying Chopin's use of these features and which thus reveal his "notational syntax". As noted, my investigation focuses only on the Barcarolle (in part because of space limitations), but it is hoped that the results will be generalisable or at least might inspire fruitful enquiry with regard to other works by Chopin.

The Barcarolle was composed between late I845 and August I846, 9 when Chopin sent the eventual French and German Stichvorlagen to Auguste Franchomme for transmission respectively to Brandus and, through Maho, to Breitkopf\&Härtel. At around the same time, Auguste Léo was entrusted with a third manuscript destined for Wessel in London. ${ }^{\text {IO }}$ The Brandus Stichvorlage - referred to as "AI" in the following discussion - ob-

9 Chopin's letter of I2 [to 26] December I845 to his family in Warsaw states: "Now I would like to finish the Cello Sonata [Op. 65], a Barcarolle [Op. 60], and some other thing that I am not certain what I will call [that is, the Polonaise-Fantasy Op. 61]". Chopin's Polish Letters, trans. by David Frick, Warsaw 2016, p. 385 .

10 In a letter of 30 August 1846 to Léo in Paris, Chopin wrote: “j'ai pensé porter les manuscrits à Paris, mais la belle saison me retient encore, et je viens vous ennuyer avec mon envoi pour Londres". " $I$ had intended to take my manuscripts to Paris but the beautiful weather is keeping me here, so I will bore you with the dispatch for London".) Correspondance de Frédéric Chopin, ed. by Bronislas Édouard Sydow with Suzanne and Denise Chainaye, Paris 198I, Vol.3, p. 239; my translation. On the same day he wrote to Wojciech Grzymała in Paris: "I am sending you a huge package for Léo - deliver it to him personally; they are manuscripts for London - I wouldn't want them to get lost". Chopin's Polish Letters, p. 392. Finally, Chopin's letter of I3 September I846 to Auguste Franchomme in Paris states: "Je suis bien fâché de ce que Brandus soit absent et que Maho ne soit pas encore en mesure de recevoir les manuscrits qu'il m'a si souvent demandés cet hiver. Il faut donc attendre - cependant je te prie d'avoir l'obligeance d'y retourner le plus tôt que tu jugeras possible - car je ne voudrais pas maintenant que cette affaire trâne en longueur, ayant envoyé ma copie à Londres en même temps qu'à toi". ("I am furious that Brandus is away and that Maho is not yet able to receive the manuscripts that he often asked me for last winter. It is therefore necessary to wait - although I would be grateful if you could try again as soon as you consider it possible to do so - because I would not like the matter to drag on, having sent my copy to London at the same time as to you".) Correspondance de Frédéric Chopin, Vol.3, p. 24I; my translation. The Breitkopf\&Härtel receipt is dated "I9. XI. 46", and the Wessel receipt "20. IX. 46"; see Kallberg: Chopin at the Boundaries, pp. 187 and 205. 
viously served as a working manuscript. The Wessel Stichvorlage - hereafter "[A2]" appears to have been prepared on the basis of AI for reasons that will become clear, although conjecture is required as it is now lost (hence the square brackets). ${ }^{I I}$ The Breitkopf manuscript - "A3" - could have been prepared in a number of ways, as we shall see. ${ }^{\text {I2 }}$ As for the first editions, the Wessel print (plate number 63I7; hereafter " $\mathrm{E}$ ") was registered on 7 October I846, whereas the Brandus edition (plate number 4609; "F") was legally deposited on I3 November I846. An advertisement in the Allgemeine musikalische Zeitung No. 46 indicates that the Breitkopf print (plate number 7545; "G") appeared in November I846. 13

It is not only interesting but methodologically significant with regard to the comparative exercise that follows to contemplate the order in which the three autograph Stichvorlagen were prepared. In the absence of [A2] this cannot be definitively determined, but internal evidence points to the first of the hypothetical filiations shown in Figure I. Both it and the second filiation indicate a two-stage process in which the later copies were prepared in direct succession, the third of them being based on the manuscript immediately preceding it rather than a common source. In contrast, filiations 3 and 4 show a single common source giving rise, in succession, to two later copies which in principle had no direct influence on one another. Filiation 5 reflects the more or less simultaneous preparation of the two later copies from one source (whether bar by bar or line by line, or resulting from some other mode of production), while the last scenario is the most flexible but, arguably, the least useful. Although it would explain all the divergences among the three different sources, the most likely filiation remains the first in Figure $\mathrm{I}^{\mathrm{I}}{ }^{4}$ The ensuing study demonstrates this on the basis of close comparison of

Because [A2] is lost, the Wessel print " $\mathrm{E}$ " replaces it in the comparative exercise that follows, despite the likelihood that E diverged from its Stichvorlage in multiple respects. Nevertheless, the care taken by the English publisher with Chopin's manuscripts was greater than is often assumed, and thus a rough-and-ready substitution of E for [A2] seems justifiable. For discussion, see Christophe Grabowski: Wessel's Complete Collection of the Compositions of Frederic Chopin. The History of a Title Page, in: Early Music 29/3 (200I), pp. 424-433; and Grabowski/Rink: Annotated Catalogue of Chopin's First Editions, PP. LII-LIX.

The Brandus manuscript, AI, is held by the Biblioteka Jagiellońska in Cracow (PL-Kj: Muz. Rkp. 2204), available at https://jbc.bj.uj.edu.pl/dlibra/publication/I732/edition/933, and the Breitkopf Stichvorlage, A3, by the British Library in London (GB-Lbl: Zweig Ms. 27), available at www.bl.uk/manu scripts/Viewer.aspx?ref=zweig_ms_27_foorr (all sites in this essay last accessed 2 February 20I9). See Grabowski/Rink: Annotated Catalogue of Chopin's First Editions, pp. 423-425; see also www.chopin online.ac.uk/aco/catalogue/barcarolle-opus-6o. Digital images of the three first editions are available at www.chopinonline.ac.uk/cfeo.

The apparent certainty with which the editors of the Wydanie Narodowe regard filiation I as definitive could inhibit a more nuanced understanding of the ways in which these sources evolved, not to mention their content and musical import; see the source commentary in Fryderyk Chopin: Dzieła Różne, 


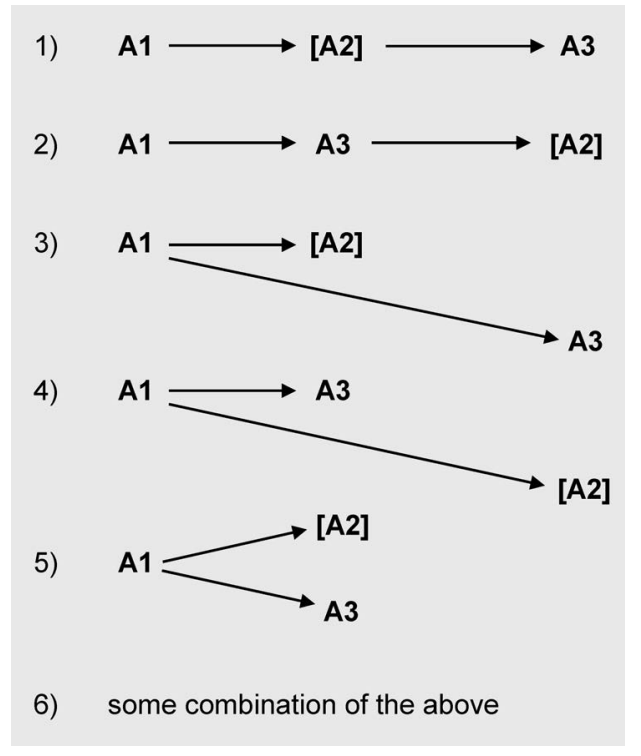

FIGURE I Possible filiations

of the Stichuorlagen for Chopin,

Barcarolle Op. 60

the three sources, revealing particularly abundant commonalities between AI and [A2] (but not A3) and between [A2] and A3 (but not AI), as well as a host of conundrums which cannot be so easily explained, among them certain parallelisms between AI and A3 (but $\operatorname{not}[\mathrm{A2}])$.

The comparison was undertaken in four phases: AI and A3; AI and E; E and A3; and AI and F. ${ }^{\text {I5 }}$ Attention was directed to seven parameters in particular, including layout (discussed below with regard to Table I); pitch notation, including rhythm (Table 2); articulation, including expression marks (Table 3); pedalling (Table 4); slurring (Table 5); dynamics (Table 6); and long accents/diminuendos (Table 7). ${ }^{16}$ In this respect and others the exercise was by no means exhaustive, but it nevertheless proved revelatory with regard to Chopin's copying practices as well as the order in which the three manuscripts were prepared.

Comparison of the page layouts across the two extant sources (shown in Table I) and of the discrepant pitch and rhythmic notation across AI, E and A3 (Table 2) sheds light on

ed. by Jan Ekier and Paweł Kamiński, Warsaw 2002 (Wydanie Narodowe, Vol. A:XII), pp. I6-20; cf. their earlier, significantly different edition of the Barcarolle published in 1992 in the same series.

15 Comparison with $\mathrm{G}$ was not undertaken, given that Chopin is likely to have had no involvement in its production once the relevant Stichvorlage had been dispatched.

16 In the tables, "note" is abbreviated "nt.", with "nts" for the plural. The appearance of + or - after a beat or note number indicates the positioning of a given feature either slightly after or before the event in question. Thus, with regard to code Artis in Table 3, the "poco più mosso" in bar 35 of source AI begins just after the fourth quaver, hence the "4+". For discussion of Chopin's long accents and other notational idiosyncrasies in the sources for the two Concertos see John Rink: Les Concertos de Chopin et la notation de l'exécution, in: Frédéric Chopin, interprétations, ed. by Jean-Jacques Eigeldinger, Geneva 2005, pp.69-88. 


\begin{tabular}{|c|c|c|c|c|}
\hline Page & System & $\begin{array}{c}\text { A1: } \\
\text { Brandus }\end{array}$ & $\begin{array}{c}\text { [A2]: } \\
\text { Wessel }\end{array}$ & $\begin{array}{c}\text { A3: } \\
\text { Br\&H }\end{array}$ \\
\hline \multirow[t]{4}{*}{1} & 1 & bar 1 & $?$ & bar \\
\hline & 2 & 6 & ? & 6 \\
\hline & 3 & 11 & $?$ & 10 \\
\hline & 4 & 16 & $?$ & 14 \\
\hline \multirow[t]{4}{*}{2} & 1 & 20 & $?$ & 18 \\
\hline & 2 & 23 & $?$ & 21 \\
\hline & 3 & 27 & $?$ & 24 \\
\hline & 4 & 30 & $?$ & 28 \\
\hline \multirow[t]{4}{*}{3} & 1 & 33 & $?$ & 32 \\
\hline & 2 & 37 & $?$ & 36 \\
\hline & 3 & 42 & $?$ & 41 \\
\hline & 4 & 47 & $?$ & 46 \\
\hline \multirow[t]{4}{*}{4} & 1 & 52 & $?$ & 51 \\
\hline & 2 & 56 & $?$ & 56 \\
\hline & 3 & 61 & $?$ & 61 \\
\hline & 4 & 66 & $?$ & 66 \\
\hline \multirow[t]{4}{*}{5} & 1 & 70 & $?$ & 71 \\
\hline & 2 & 74 & $?$ & 76 \\
\hline & 3 & 78 & $?$ & 81 \\
\hline & 4 & 82 & $?$ & 85 \\
\hline \multirow[t]{4}{*}{6} & 1 & 86 & $?$ & 90 \\
\hline & 2 & 90 & $?$ & 93 \\
\hline & 3 & 93 & $?$ & 97 \\
\hline & 4 & 97 & $?$ & 101 \\
\hline \multirow[t]{4}{*}{7} & 1 & 101 & $?$ & 105 \\
\hline & 2 & 105 & $?$ & 109 \\
\hline & 3 & 110 & $?$ & 112 \\
\hline & 4 & 113 & $?$ & 115 \\
\hline
\end{tabular}

TAB LE I Page layouts in the autograph Stichvorlagen of Chopin, Barcarolle Op. 60

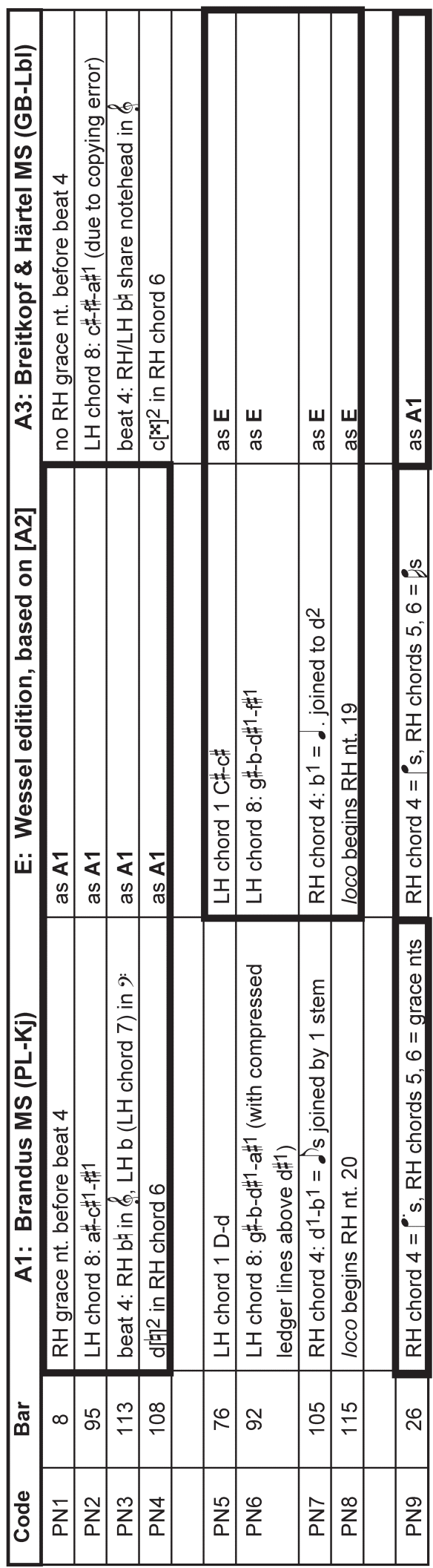


what one might call the "skeleton" of the music, as opposed to the constituent articulation marks and other expressive indications discussed later. Although little insight can be gleaned about Chopin's notational practices and predilections from the respective layouts, they reveal a fairly close adherence to his original source across the filiation chain. Understandably, where AI contains extensive deletions or insertions, the page layout in the later A3 changes commensurately - and presumably this also happened when [A2] was prepared.

The pitch material sheds greater light on compositional decision-making and notational method. Although there is evidence of miscopying, Chopin closely followed the progenitor source(s) on the whole. Moreover, individual pitches that remain on their original staves from copy to copy tend to retain their notational appearance - for instance, with regard to stem direction - although some variability is revealed concerning the staff on which given pitch material appears (either treble or bass) and also the use of ottava signs. Note in particular the errors in pitch notation in bars 92 and 95 (respectively codes PN6 and PN2 in Table 2); ${ }^{77}$ the different pitch identified in bar 76, which possibly resulted from an erroneous reading on Chopin's part (PN5); the changes in rhythmic value in bars 26 and I05, also perhaps the result of error (respectively PN9 and PN7); and certain notational alternatives with regard to placement on the Grand Staff(PN3), positioning under an ottava (PN8) and pitch orthography (PN4), at least some of which might reflect the order in which the later manuscripts were produced. In that respect, it is also interesting to note the common features here and indeed in all of the remaining tables between different pairs of sources - AI and E, E and A3, AI and A3 - of which the last set of commonalities is less easy to explain given the order of preparation proposed above.

A good deal of flesh can be added to the skeleton thus outlined by comparing the discrepant articulation (including expression marks) across the three sources, as shown in Table 3. One of the most interesting instances is found in bar 83 (code Art4), where, in both AI and $\mathrm{E}$, the ritenuto starts from right-hand ( $\mathrm{RH}$ ) note I9 and appears above the music, whereas in $\mathrm{A}_{3}$ it starts six notes earlier and is written underneath the $\mathrm{RH}$ part. Whether this reflects a deliberate change of mind, an "equating" of the two positionings or simply an error is impossible to determine, although in general I have observed the following tendencies in Chopin's treatment of articulation and expression marks: frequent omission, ongoing emendation, and a flexible approach to the centring (or not) of the marks in question within the bar or on the relevant figuration, the last of which

17 See the explanation in John Rink: The Barcarolle. Auskomponierung and Apotheosis, in: Chopin Studies, ed. by Jim Samson, Cambridge I988, pp. I95-220, here pp. 208 f., notes I2 and I3. Here and throughout this discussion readers will find it useful to consult the two manuscripts cited in note I2 above and the English first edition at www.chopinonline.ac.uk/cfeo. 


\begin{tabular}{|c|c|c|c|c|c|c|c|c|c|c|c|c|c|c|c|c|c|c|c|c|c|}
\hline $\begin{array}{l}: \pi \\
\text { :T } \\
\infty\end{array}$ & 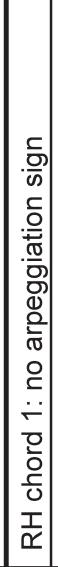 & 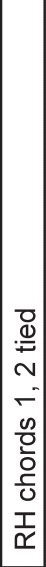 & 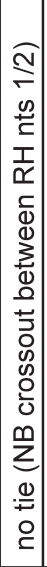 & 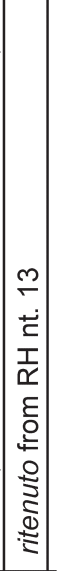 & $\begin{array}{l}\boldsymbol{w} \\
\mathfrak{\sigma} \\
\widetilde{\sigma}\end{array}$ & 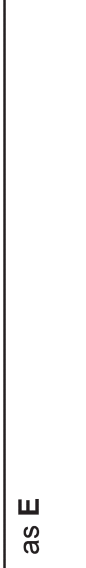 & $\begin{array}{l}\boldsymbol{w} \\
\mathbb{D} \\
\mathbb{\sigma}\end{array}$ & 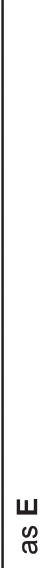 & \begin{tabular}{|l}
$w$ \\
w
\end{tabular} & 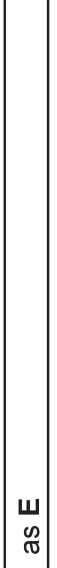 & \begin{tabular}{|l|}
$\mathbf{w}$ \\
$\mathscr{\tau}$
\end{tabular} \mid & 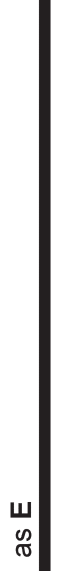 & $\begin{array}{c}\bar{\tau} \\
0 \\
\mathbb{\sigma}\end{array} \mid$ & 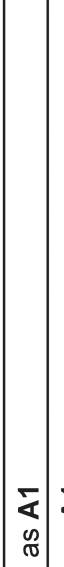 & $\mid$ & 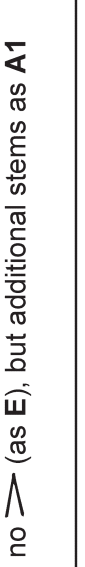 & $\begin{array}{l}\bar{\alpha} \\
\tilde{q} \\
\tilde{\sigma}\end{array}$ & 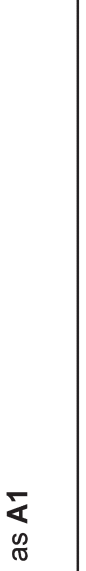 & & & 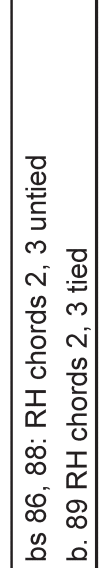 \\
\hline 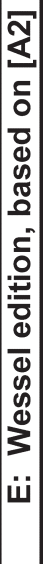 & 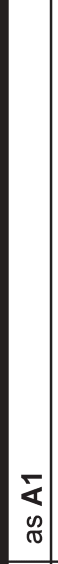 & $\left|\begin{array}{c}\bar{\alpha} \\
0 \\
\pi\end{array}\right|$ & $\mid \begin{array}{l}\tau \\
\mathbb{\alpha} \\
\sigma \\
\sigma\end{array}$ & \begin{tabular}{|l|}
$\bar{\alpha}$ \\
0 \\
$\pi$
\end{tabular} & 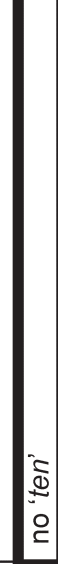 & 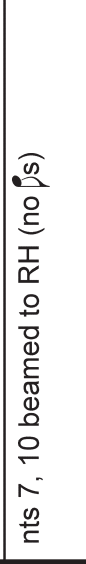 & 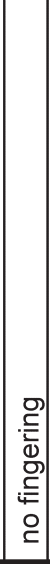 & 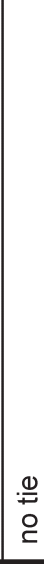 & $\mid$ & 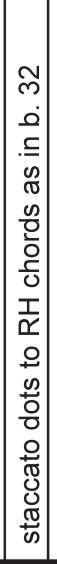 & 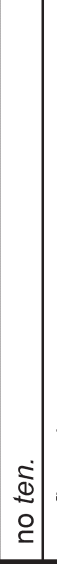 & 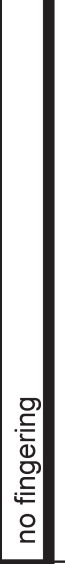 & $\mid \begin{array}{l}- \\
0 \\
0 \\
\frac{c}{0} \\
0 \\
\frac{1}{\alpha x} \\
0 \\
0 \\
0 \\
0 \\
0 \\
0 \\
0 \\
0 \\
\frac{\pi}{0} \\
0 \\
0 \\
c\end{array}$ & 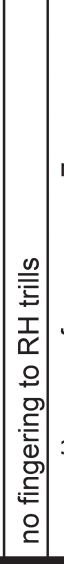 & 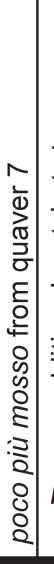 & 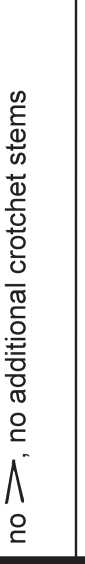 & 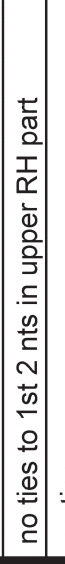 & 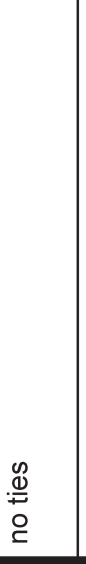 & 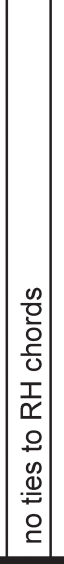 & 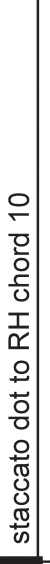 & 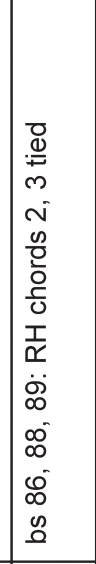 \\
\hline 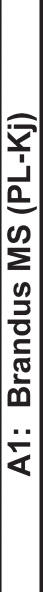 & 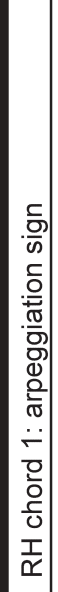 & 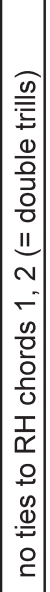 & 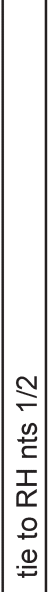 & 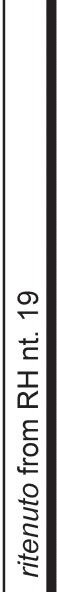 & 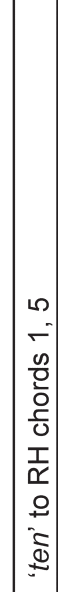 & 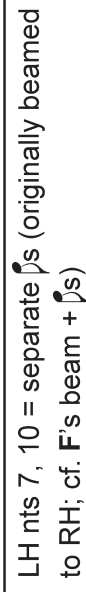 & 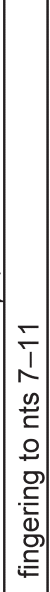 & 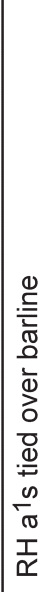 & 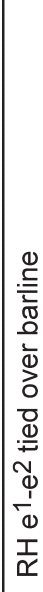 & 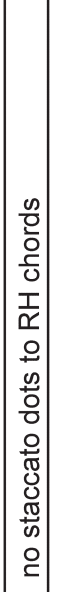 & 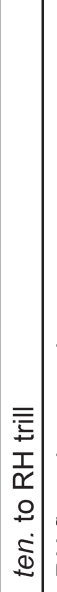 & 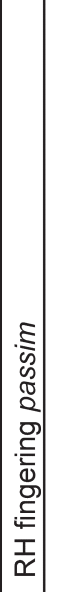 & 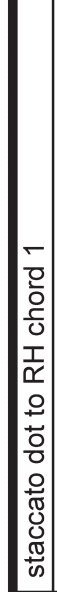 & 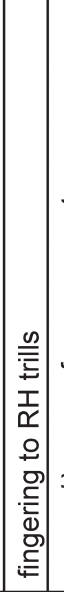 & 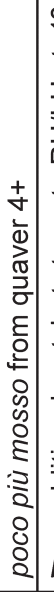 & 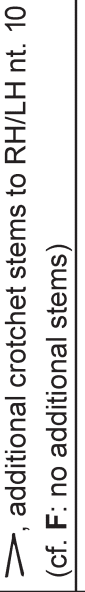 & 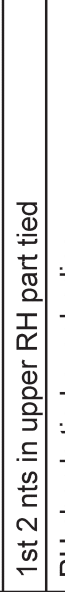 & 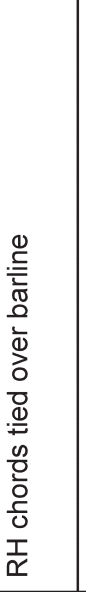 & 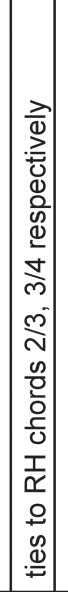 & 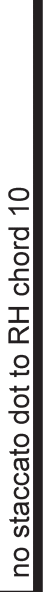 & 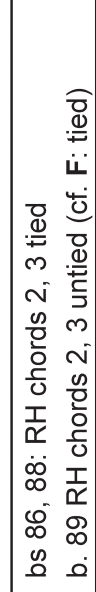 \\
\hline Фొ & $\infty$ & $\stackrel{\sim}{\sim}$ & 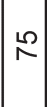 & $\begin{array}{l}m \\
\infty\end{array}$ & $\bar{m}$ & 禺 & 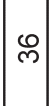 & $\frac{9}{\partial}$ & 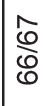 & ๙ & $\stackrel{ }{\circ}$ & 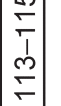 & $\approx$ & $\left|\begin{array}{l}\mathbb{N} \\
\tilde{N} \\
N\end{array}\right|$ & 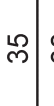 & 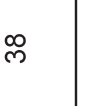 & 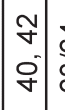 & 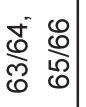 & $\mid \begin{array}{l}0 \\
0 \\
0 \\
0 \\
0\end{array}$ & & $\begin{array}{l}\infty \\
\infty \\
\infty \\
\infty \\
\infty\end{array}$ \\
\hline ¿ू & $\overline{\frac{E}{\alpha}}$ & $\stackrel{2}{\frac{1}{\alpha}}$ & $\frac{t}{<}$ & $t$ & $\mid \begin{array}{l}\frac{6}{2} \\
\frac{1}{4}\end{array}$ & $\mid \begin{array}{l}0 \\
\frac{1}{2}\end{array}$ & & 紊 & 昰 & 呈 & $\frac{t}{4}$ & $\begin{array}{l}\frac{N}{ \pm} \\
\frac{T}{\alpha}\end{array}$ & $\frac{m}{\frac{m}{\alpha}}$ & $\mid \begin{array}{l} \pm \\
\\
\frac{E}{4}\end{array}$ & $\stackrel{2}{\frac{2}{2}}$ & $\frac{7}{4}$ & $\begin{array}{l}\hat{E} \\
\bar{E}\end{array}$ & $\sum_{\frac{\pi}{2}}^{\infty}$ & $\mid \frac{9}{\frac{O}{4}}$ & & 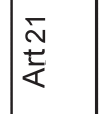 \\
\hline
\end{tabular}

TABLE 3 Articulation 


\begin{tabular}{|c|c|c|c|c|c|c|c|c|c|c|c|c|c|c|c|c|c|c|c|}
\hline & $\begin{array}{l} \\
\\
\oplus \\
\circ \\
\circ\end{array}$ & 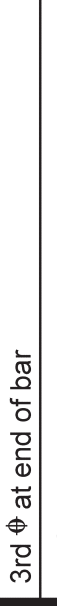 & 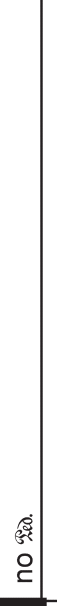 & $\left|\begin{array}{l}w \\
g \\
\tau\end{array}\right|$ & $\begin{array}{l}w \\
\tilde{\sigma} \\
\tilde{\sigma}\end{array}$ & \begin{tabular}{|l|}
$w$ \\
$\tilde{\sigma}$ \\
\end{tabular} & $\begin{array}{l}\underset{\sigma}{g} \\
\sigma\end{array}$ & $\begin{array}{l}\underset{w}{g} \\
\sigma\end{array} \mid$ & $\begin{array}{l}\underset{w}{w} \\
\tilde{\sigma}\end{array} \mid$ & $\underset{\substack{\infty \\
\varpi}}{w_{0}}$ & $\begin{array}{c}w \\
\tilde{g} \\
\tilde{\sigma}\end{array}$ & 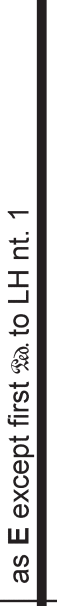 & \begin{tabular}{|l}
$\bar{\Sigma}$ \\
$\mathscr{c}$ \\
$\tilde{\sigma}$
\end{tabular} & 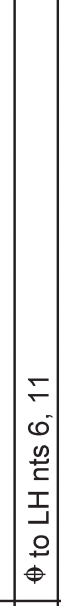 & 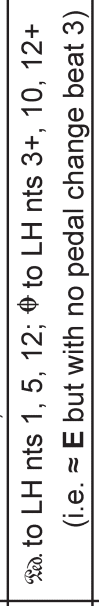 & 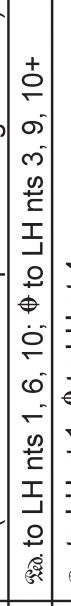 & 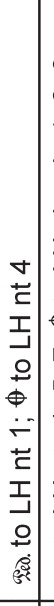 & 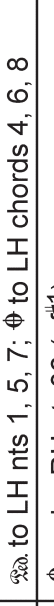 & 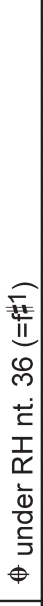 \\
\hline 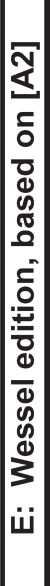 & 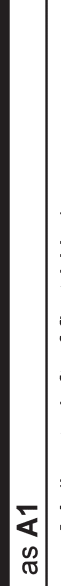 & 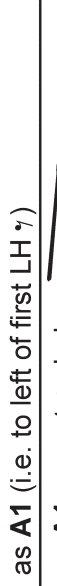 & 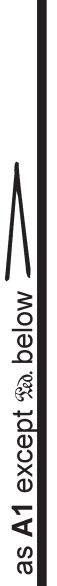 & 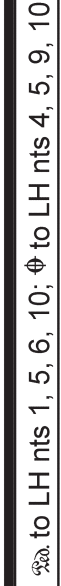 & 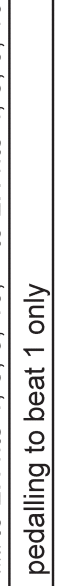 & 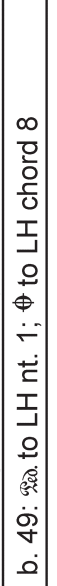 & 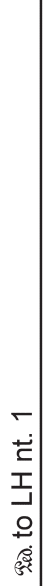 & 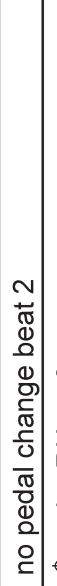 & 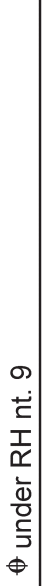 & 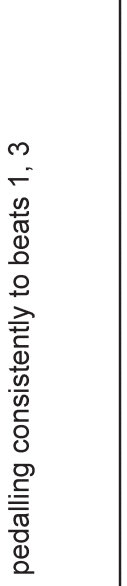 & 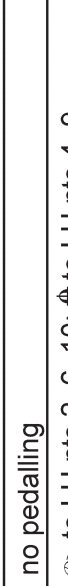 & 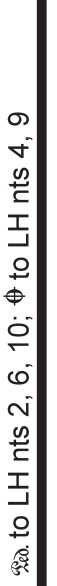 & 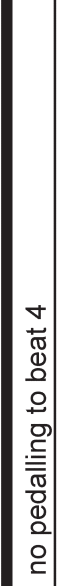 & 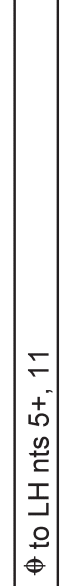 & 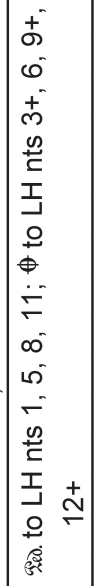 & 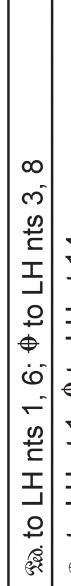 & 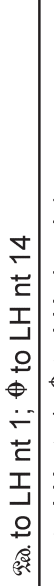 & 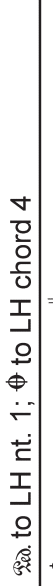 & 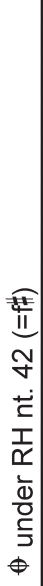 \\
\hline 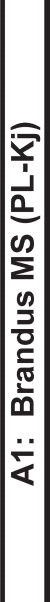 & 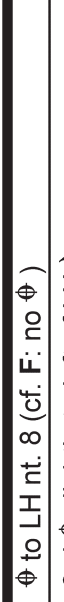 & 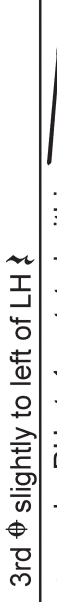 & 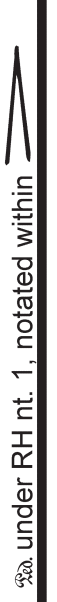 & 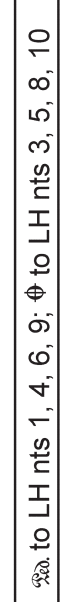 & 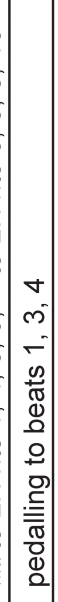 & 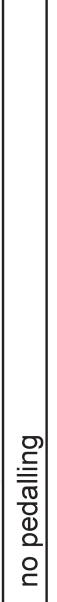 & 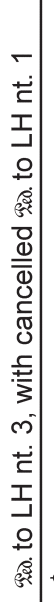 & 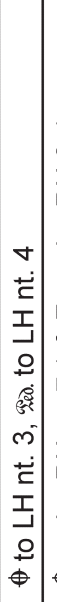 & 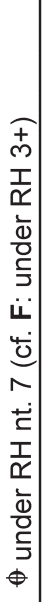 & 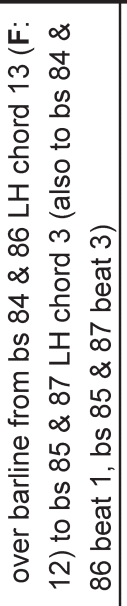 & 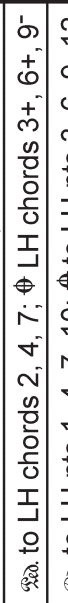 & 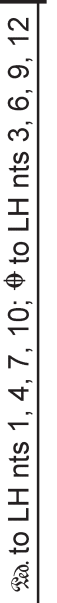 & 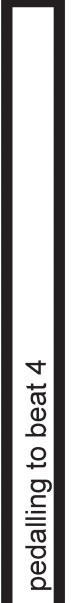 & 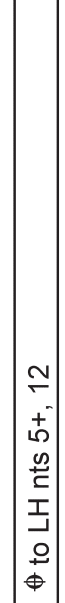 & 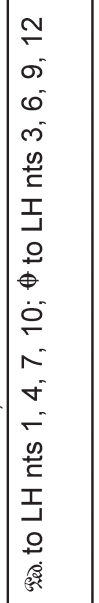 & 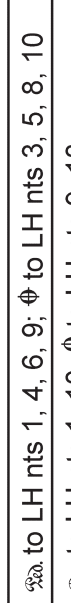 & 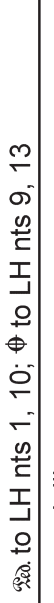 & 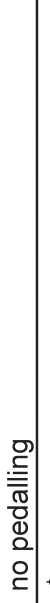 & 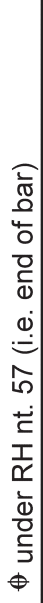 \\
\hline 商 & 员 & $\bar{\sigma}$ & $\nwarrow$ & $\stackrel{20}{2}$ & $\hat{N}$ & g & ల్రి & $\mid \begin{array}{l}\infty \\
0\end{array}$ & $\stackrel{\infty}{\sim}$ & 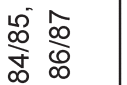 & $\stackrel{\nabla}{=}$ & 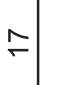 & $\stackrel{N}{~}$ & 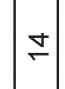 & $\stackrel{0}{\circ}$ & m) & స్ & $\stackrel{\infty}{\sim}$ & $\stackrel{10}{\digamma}$ \\
\hline $\begin{array}{l}0 \\
0 \\
0 \\
0 \\
0\end{array}$ & $\bar{\alpha}$ & $\mathbb{\alpha}$ & $a$ & $\mid \begin{array}{l}0 \\
\mid a\end{array}$ & 0 & ○ & $\hat{a}$ & $\begin{array}{l}\infty \\
0\end{array}$ & ○ & & $\bar{\Sigma}$ & $\bar{N}$ & & $\frac{\sigma}{\alpha}$ & $\frac{1}{\alpha}$ & $\frac{0}{2}$ & $\overline{0}$ & $\frac{\infty}{\square}$ & $\frac{\sigma}{\alpha}$ \\
\hline
\end{tabular}

TABLE 4 Pedalling 
constitutes a general "problem" posed by Chopin's notation. These tendencies can be seen by reviewing the examples in Table 3 and comparing the sources in question.

Study of the pedalling indications is particularly interesting not least because of the many variants (for example, Pio in Table 4) that characterise these and other late sources such as the manuscripts and first editions of the Polonaise-Fantasy Op. 61. Some changes do not seem as considered as others, however. Indeed, Table 4 reveals four notational tendencies on Chopin's part of which he was probably unaware. These concern a general rightward shift of pedal marks and pedal releases (see for example P4, P9, PI5 and PI7); the removal of pedalling (P5, P8, PII, PI2, PI5 and PI7); conversely, the addition of pedalling (P6, PI6 and PI8), which happens relatively rarely as against a trend towards less rather than more pedalling; and a repositioning of pedal releases, which, as in bar 6I (P2), tend to move to the right during the copying process. For the reasons given earlier, all of these need to be viewed as possible habits affecting the source material of other works which Chopin "copied" in one or more of the senses that I have defined. The position of, say, a given pedal mark or pedal release should be evaluated with regard to the status of the source or of the given notation within the filiation in question, in addition to judging it within its immediate context.

Slurring tends to remain stable across select pairs of sources (generally AI and E, or $\mathrm{E}$ and $\mathrm{A} 3$ ), but the position thereof can move either above or below (or both) with reference to the figuration in question. Similarly, the beginning and end points of slurs can change significantly. We also find that presumed notational inaccuracies inadvertently become the basis of new "legitimated" but essentially erroneous readings in later sources. This is a characteristic of the printed sources too, whether across successive impressions of the first editions or in subsequent editions. Table 5 reveals some apparent notational alternatives - among them those in bars IO-I2 (SI), 78-79 (s5) and 6-8 (s9), the last involving two RH slurs in AI (the second of which is related to an inner part in bars 6-7) as against the seemingly equivalent overarching RH slur in both $\mathrm{E}$ and $\mathrm{A}$. Changes in content possibly arising from notational inexactitude comprise the following: S2 (where the RH slur starts from RH chord I in AI and E, but from RH chord 2 in A3), SI5 (where AI contains no RH slur, E has a RH slur like that in bar 32, and A3 also has a RH slur which is shifted slightly to the right, as if from RH chord 2) and s7 (concerning the occasionally shortened LH slur patterns in AI, which give rise to consistently abbreviated slurring in A3 though not in E). One can also infer two typical practices from the discrepancies shown in Table 5: the breaking of long slurs into two or more short(er) slurs (SI, S4, S5, SI2, SI3, SI7 and S25), and the formation of long slurs from successive shorter ones (s6, s8, s9 and SII). It is almost as if Chopin chooses to adopt a policy of opposition here - in other words, as if his creative impulse results in a new reading not just different from but literally opposed to the original one. 


\begin{tabular}{|c|c|c|c|c|c|c|c|c|c|c|c|c|c|c|c|c|c|c|c|c|c|}
\hline 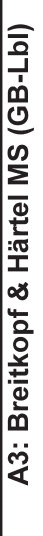 & 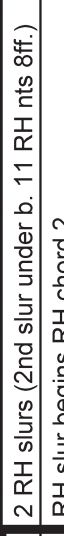 & 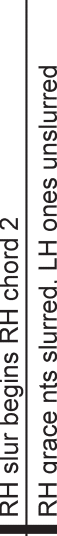 & 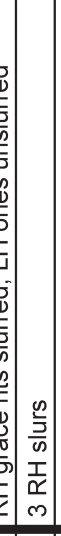 & 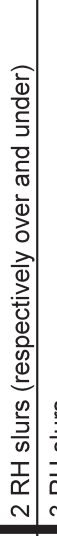 & & 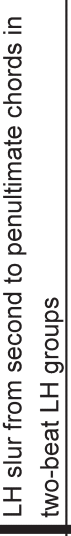 & 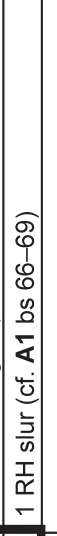 & \begin{tabular}{|l|} 
\\
$\mathbb{w}$ \\
$\approx$ \\
\end{tabular} & \begin{tabular}{|l}
$\mathbf{w}$ \\
$\mathscr{D}$ \\
$\varpi$
\end{tabular} & 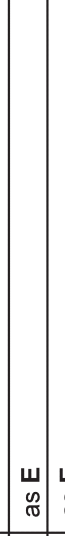 & \begin{tabular}{|c|}
$\mathbf{w}$ \\
$\mathscr{c}$ \\
$\boldsymbol{\sigma}$
\end{tabular} & 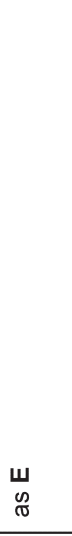 & \begin{tabular}{|l}
$w$ \\
$\mathscr{g}$ \\
$\tilde{\sigma}$
\end{tabular} & 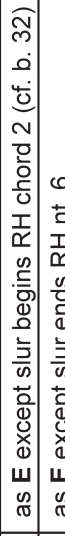 & 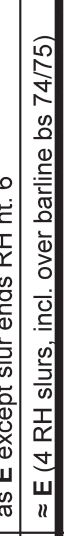 & 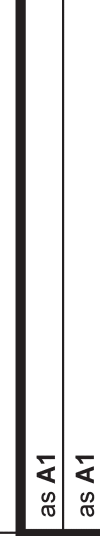 & 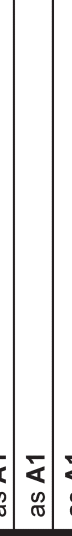 & 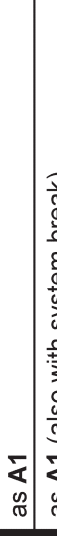 & 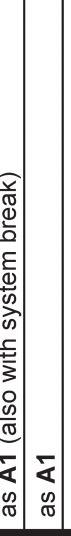 & 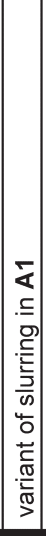 & 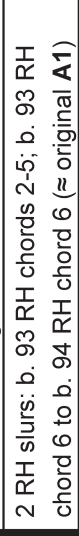 \\
\hline 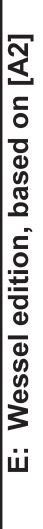 & 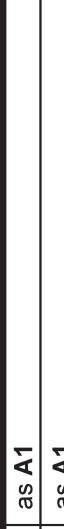 & & 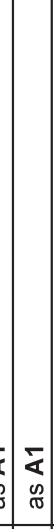 & 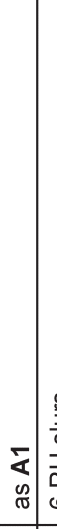 & 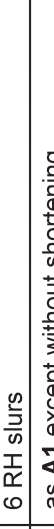 & 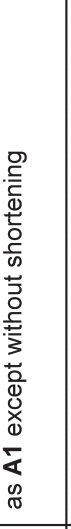 & 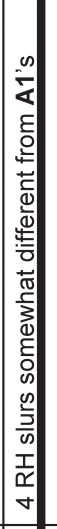 & 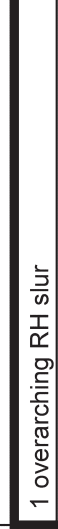 & 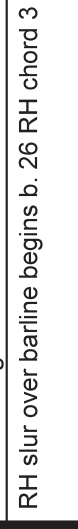 & 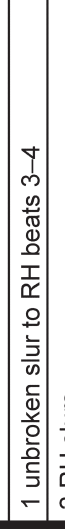 & | & 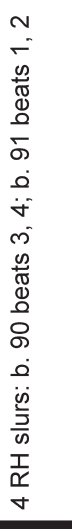 & 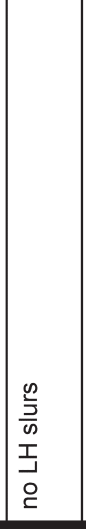 & 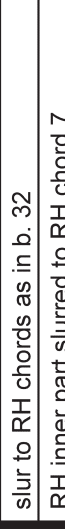 & 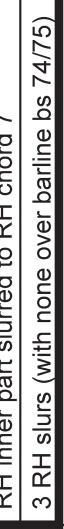 & 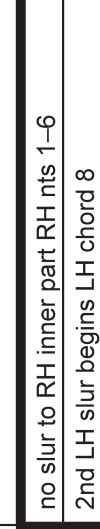 & 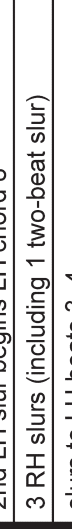 & 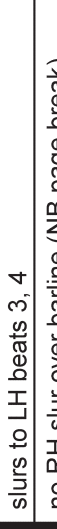 & 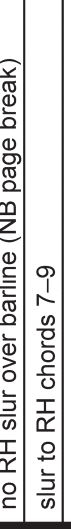 & & 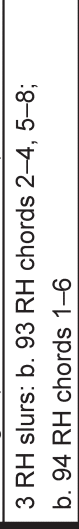 \\
\hline 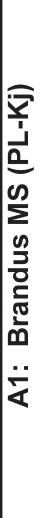 & 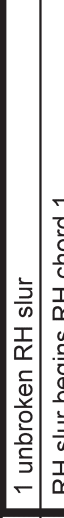 & 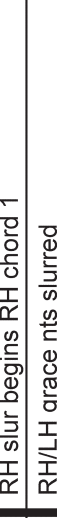 & 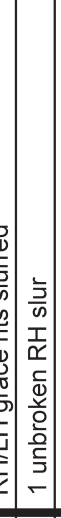 & 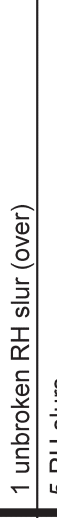 & 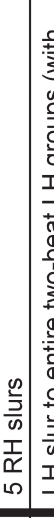 & 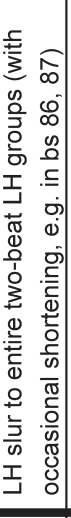 & $\mid$ & 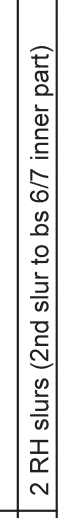 & 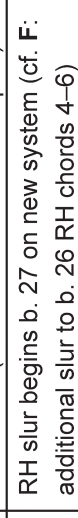 & 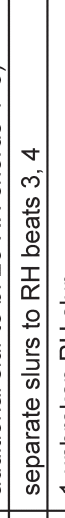 & 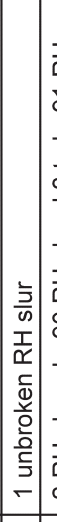 & 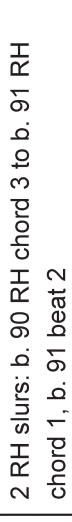 & 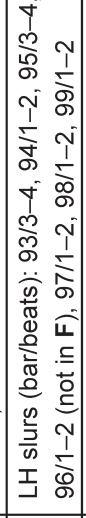 & 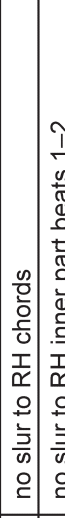 & 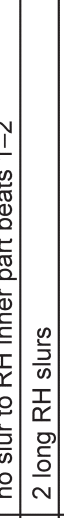 & 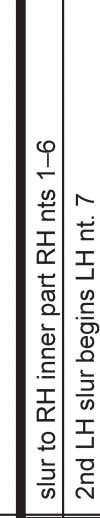 & 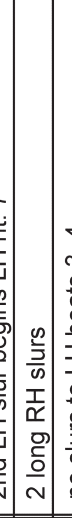 & 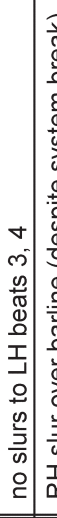 & 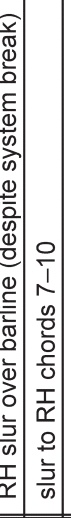 & & 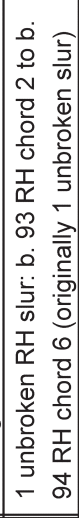 \\
\hline ळ̄ & \begin{tabular}{c}
$\mathrm{N}$ \\
\multirow{1}{c}{} \\
\hdashline
\end{tabular} & ") & $\mid \begin{array}{l}0 \\
\hat{p} \\
\frac{1}{5} \\
\end{array}$ & 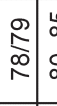 & \begin{tabular}{c|c}
$\infty$ & \\
$\infty$ & \\
1 & \\
$\infty$ & $\delta$
\end{tabular} & 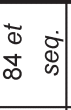 & \begin{tabular}{|l|} 
\\
$\frac{1}{1}$ \\
6 \\
\end{tabular} & $\begin{array}{l}0 \\
0 \\
0\end{array}$ & $\begin{array}{l}\mathfrak{N} \\
\stackrel{N}{N}\end{array}$ & $\bar{m}$ & \begin{tabular}{|l|l} 
& \\
1 & \\
1 & \\
0 & \\
\end{tabular} & চे & $\begin{array}{l}8 \\
0 \\
0 \\
1 \\
\infty\end{array}$ & $\approx$ & 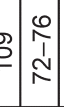 & $=\bar{N}$ & 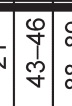 & \begin{tabular}{l|l}
8 & \\
$\infty$ & $\delta$ \\
$\infty$ & $\delta$ \\
$\infty$ & $\delta$
\end{tabular} & 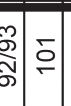 & & \begin{tabular}{|l} 
\\
O \\
O
\end{tabular} \\
\hline ס & $\bar{\omega} \delta$ & & & ம & & í & $\stackrel{\infty}{\infty}$ & ஆ & $\bar{s}$ & $\overline{\check{s}}$ & $\bar{N}$ & $\bar{\omega}$ & $\bar{c}$ & $\frac{\infty}{\infty}$ & is & \begin{tabular}{|l|l}
$\infty$ & $\infty$ \\
$\bar{c}$ & $\frac{9}{\omega s}$
\end{tabular} & م. & $\overline{\tilde{\omega}}$ & & & $\begin{array}{l}\stackrel{\mathscr{N}}{\infty} \\
\text {. }\end{array}$ \\
\hline
\end{tabular}

TABLE 5 Slurring 


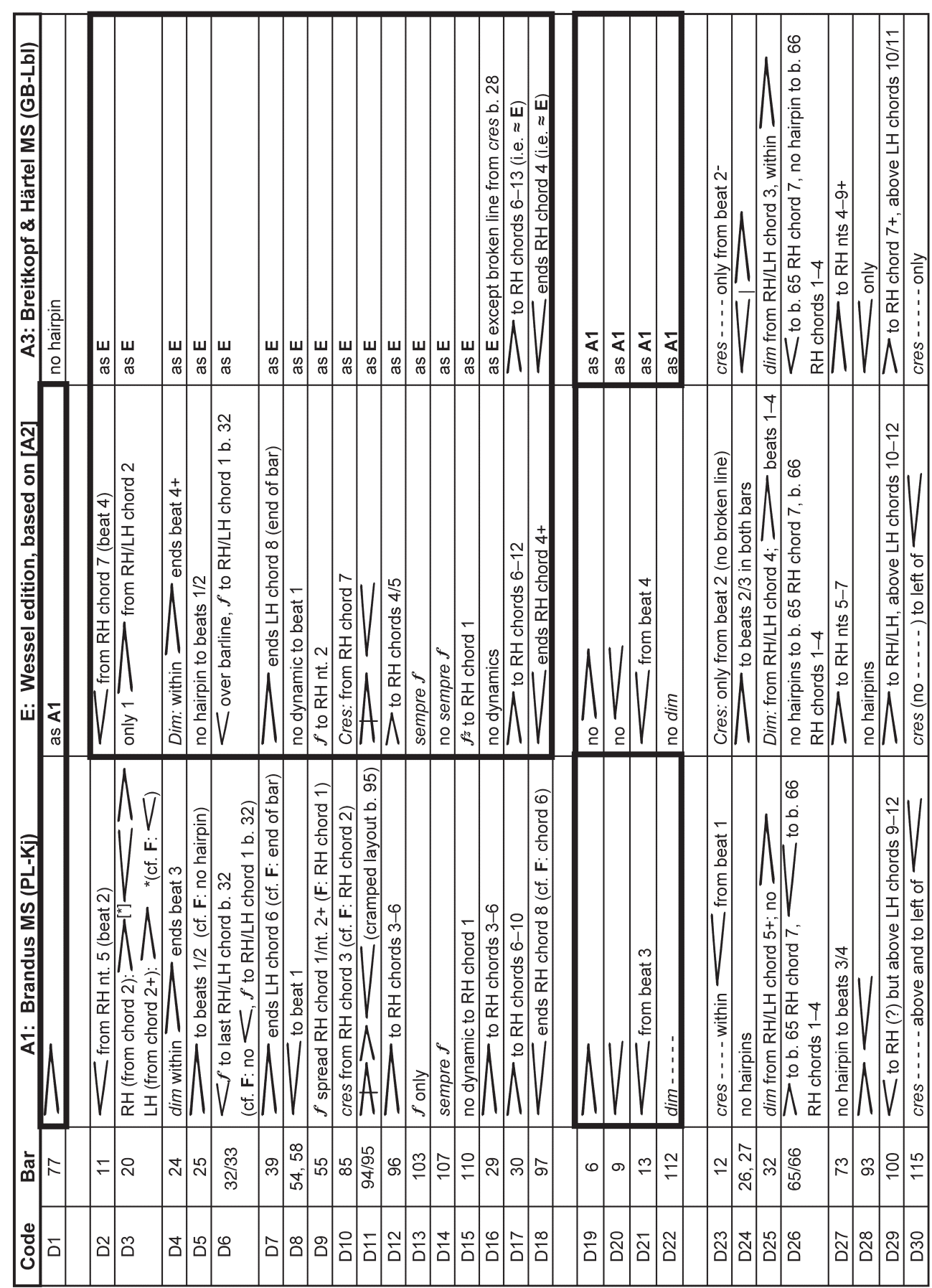

TABLE 6 Dynamics 
The considerable discrepancies among the dynamic markings within the three sources fall into several categories defined by distinct notational tendencies on Chopin's part(see Table 6). When preparing the later sources, Chopin typically lengthened hairpins (see for example D4, D7 and DI7, although compare DI2, DI8 and D23) in what amounts to a rightward extension of the symbols in question analogous to those affecting other parameters as previously described. It is also interesting to observe the relationships outlined below:

I) between RH and LH dynamics (as in the case of $\mathrm{D} 3$, where the multiple hairpins assigned to both hands in AI become amalgamated into a single $\mathrm{RH} / \mathrm{LH}$ hairpin in $\mathrm{E}$ which is retained in A3);

2) among the variable notations cres, cres — and cres - - - -, as well as cres within a hairpin; ${ }^{\text {I8 }}$

3) between symbols centred, as opposed to off-centre, either on relevant figuration or within a given bar (as in bar 32 - in other words D25 - where the "Dim:" in E and the "dim - - . -" in A3 appear in different positions not only within the bar but also with respect to an additional diminuendo hairpin which happens to be absent from AI); and

4) between the $>$ (long accent) and $<$ signs identified in D26 and D29, which reflect either a change of conception on Chopin's part or possibly an error or anomaly.

The cramped space available to Chopin in bars 94-95 of AI (DII) gave rise to two successive diminuendo hairpins which become fused in the more spaciously laid-out $\mathrm{E}$ and $\mathrm{A}$, while a different sort of notational change occurs in bar 55(D9), where the large RH/LH $\boldsymbol{f}$ spread over quavers I and 2 in AI appears cleanly under RH note 2 in $\mathrm{E}$ and $\mathrm{A} 3$ and thus does not affect the downbeat at all, possibly contrary to Chopin's intentions.

Table 7 identifies notational discrepancies forming a subset of those concerning dynamics. Here the focus is on long accents and diminuendo hairpins, one of the more arcane aspects of Chopin's notation which will be better understood by close inspection of the examples on offer. First of all, Chopin seems to have had a strong tendency to lengthen long accents in successive copying and thus, in effect, to make them look like or even become diminuendo signs. This again involves a "spreading" towards the right which can be seen in LAI, LA2, LA5, LA7, LAI4, LAI7, LAI9 and LA2O. A particularly interesting instance arises in the opening bar, where the $\boldsymbol{f}>$ to $\mathrm{RH}$ chord I in AI is

18 It has been suggested (for example, by David Kraehenbuehl: Rubato Revisited, Princeton I976, p. 6) that "nested" signs comprising cresc. or dim. within a hairpin were used by certain composers to denote tempo change rather than dynamic change. Comparison of the Barcarolle manuscripts indicates otherwise, however: see for example the discrepant notation in AI, E and A3 in bars I2 and 32 (respectively, codes D23 and D25). 


\begin{tabular}{|c|c|c|c|c|c|c|c|c|c|c|c|c|c|c|c|c|c|c|c|}
\hline & 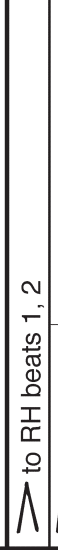 & 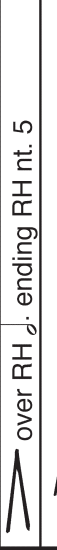 & 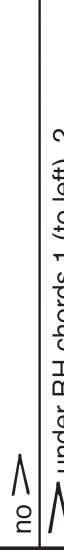 & 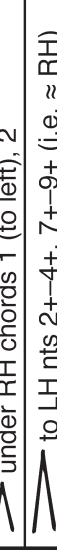 & 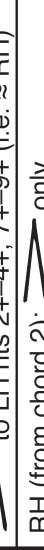 & 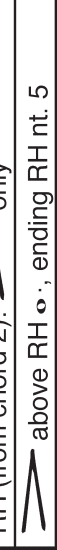 & $\left|\begin{array}{c}w \\
\omega\end{array}\right|$ & $\mid$\begin{tabular}{|c|}
$\mathbf{w}$ \\
$\boldsymbol{\Phi}$ \\
$\boldsymbol{\varpi}$
\end{tabular} & $\left|\begin{array}{|c|}\mathbf{w} \\
\widetilde{\tau} \\
\approx\end{array}\right|$ & \begin{tabular}{|c|}
$w$ \\
$\mathscr{\sigma}$ \\
$\tilde{\sigma}$
\end{tabular} \mid & 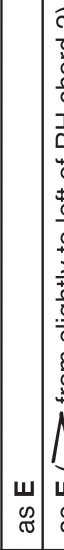 & 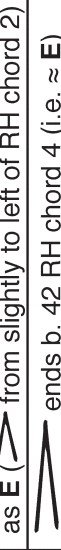 & 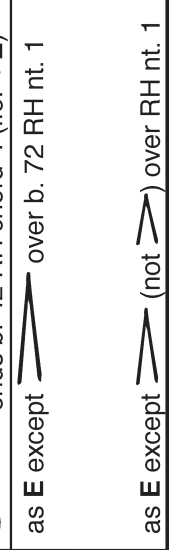 & 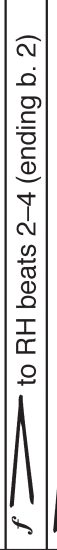 & 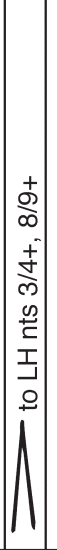 & 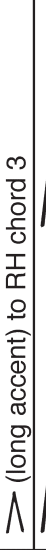 & 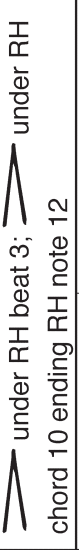 & 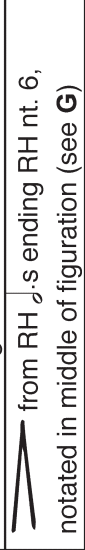 & 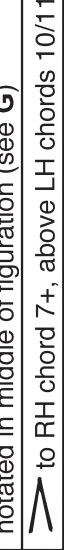 \\
\hline & $\begin{array}{l}-\pi \\
\tilde{g} \\
\tilde{\sigma}\end{array}$ & & & $\begin{array}{c}\mathbb{d} \\
\mathbb{d} \\
\mathbb{d}\end{array}$ & 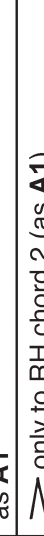 & 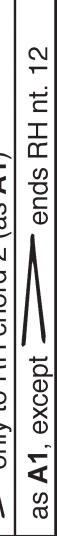 & 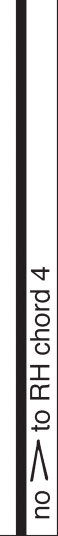 & \begin{tabular}{|l} 
\\
$\Lambda$ \\
$\vdots$ \\
$\vdots$
\end{tabular} & 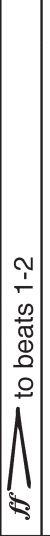 & $\begin{array}{c}0 \\
- \\
0 \\
0 \\
0 \\
0 \\
\frac{0}{0} \\
\frac{1}{\alpha} \\
0 \\
0 \\
\Lambda\end{array} \mid$ & 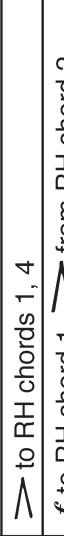 & 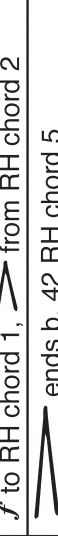 & 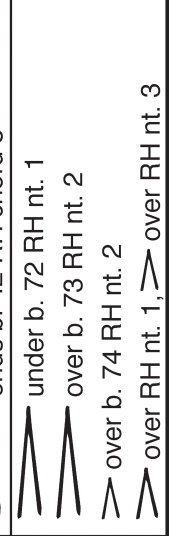 & 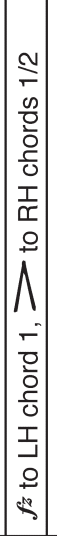 & 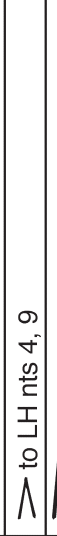 & 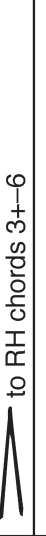 & 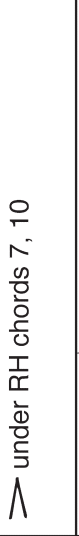 & \begin{tabular}{|l}
$\frac{0}{1}$ \\
$\frac{1}{\alpha}$ \\
$\frac{\Phi}{\omega}$ \\
0 \\
$\Lambda$ \\
$\Lambda$
\end{tabular} & 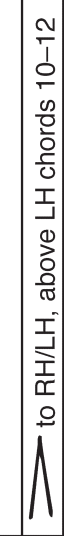 \\
\hline & $\begin{array}{l}0 \\
- \\
-0 \\
\frac{0}{0} \\
\frac{0}{0} \\
0 \\
\frac{1}{\pi} \\
0 \\
\stackrel{\Lambda}{0}\end{array}$ & 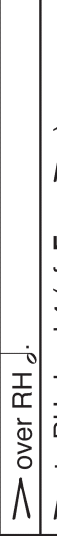 & 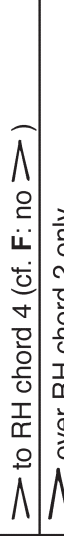 & 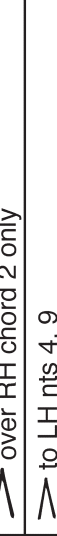 & 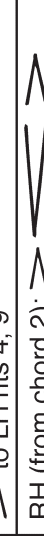 & 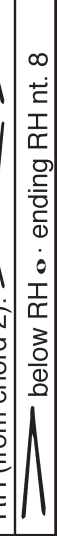 & 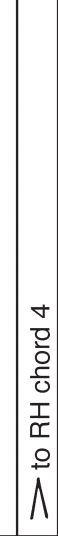 & 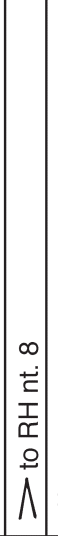 & 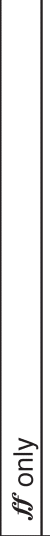 & 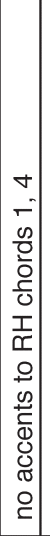 & 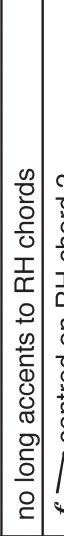 & 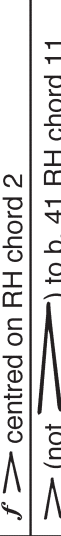 & 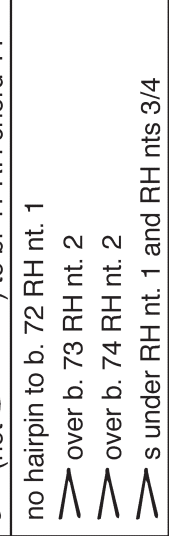 & 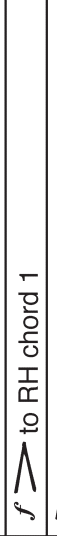 & 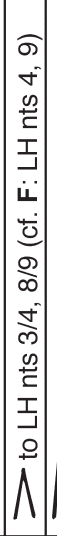 & 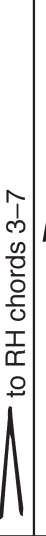 & 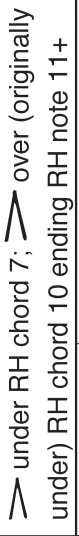 & 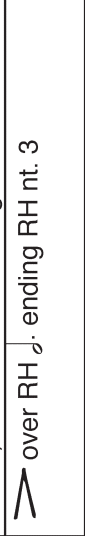 & 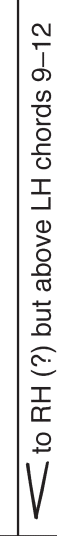 \\
\hline ๓ & $\bar{m}$ & i & $\overline{6} \delta$ & 8 & 0 & 58 & $\mp$ & 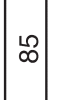 & $\delta$ & 뇽 & $\left|\begin{array}{c}0 \\
0 \\
0 \\
0 \\
0\end{array}\right|$ & $\stackrel{\nabla}{\stackrel{f}{\vec{f}}}$ & 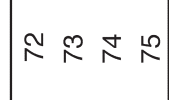 & - & 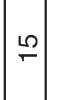 & $\stackrel{\infty}{\sim}$ & & 8 & 우 \\
\hline $\bar{z}$ & $\bar{\Xi}$ & 15 & $\stackrel{9}{5}$ & 苦 & & & $\stackrel{\infty}{\leq}$ & & & & $\frac{N}{S}$ & & 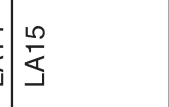 & $\left|\begin{array}{l}0 \\
\pm \\
5\end{array}\right|$ & $\frac{1}{\leq}$ & & & $\stackrel{\mathbb{N}}{\leq}$ & $\overline{\bar{y}}$ \\
\hline
\end{tabular}

TAB LE 7 Long accents/diminuendos 
transformed into two separate symbols in $\mathrm{E}-\boldsymbol{f} z$ to LH chord I and $>$ to the first three RH "events"I9 - and into $\boldsymbol{f}=$ in A3, with the hairpin stretching from just after RH chord I to the end of the bar. These differences could of course reflect a change of conception, but it is more likely that a successive stretching of the long accent occurred - one of which Chopin might not have been aware, possibly because the effect of the diminuendo sign at the end of the bar could easily have been conflated with that of the ever-expanding hairpin. One's decision as an editor here would inevitably be predicated on a host of factors, ${ }^{20}$ but the implication of this investigation is that the notation in AI reflected the composer's real intentions, whereas the general "stretching" tendency affecting Chopin's long accents in the copying process unwittingly led to the notation in A3, which thus could have a dubious status. ${ }^{2 \mathrm{I}}$

It is also interesting to note the shifting positions of long accents above or below material and in relation to the hand(s) in question (see for instance LA4, LAI5, LAI9 and LA2O), just as the identification of possible notational alternatives with regard to what one might term "conjunct long accents" is revelatory. In bar IO2, for example (LAIO), AI has $\boldsymbol{f f}$ to RH note I only (where a trill begins) as opposed to E's and A3's $\boldsymbol{f f}=$ to all of RH beats I-2 (that is the full extent of the trill); the possible equivalence of these is noteworthy and could be instructive to the editors of much earlier music by Chopin (for example, the piano concertos, where questions arise about the relationship among apparently different but possibly analogous notations such as those in parallel passages). Note also bar I4 (LAI3), where AI has $\boldsymbol{f}>$ centred on RH chord 2 (although the $\boldsymbol{f}$ actually falls under the preceding crotchet rest), E has $\boldsymbol{f}$ to RH chord I and $>$ beginning from RH chord 2, and A3 is similar to E but with the $>$ starting slightly to the left of RH chord 2. Possibly the result of misreading on Chopin's part, the separation of $\boldsymbol{f}$ and $>$ in E and A3 could have been unintentional (as argued above in the case of bar I), but it might also have arisen from notational alternatives which were equivalent in intention but potentially different in effect.

ts

As noted previously, this discussion has not aimed to be comprehensive or exhaustive with regard to Chopin's notational practice, but if nothing else the conclusions outlined

19 Here it is impossible to determine whether the English publisher conformed to Chopin's notation in [A2] or introduced a different reading of his own.

20 Interestingly, the 1992 edition of the Barcarolle in the Wpdanie Narodowe invents an altogether new reading here, assigning the $\boldsymbol{f}$ to LH chord I but extending the hairpin across all of beats 2 and 3 - thus deviating from any of the original sources. In contrast, the putative "Urtext" published in the Wpdanie Narodowe in 2002 conforms to AI, at least in this respect.

21 Compare the discussion in the Wydanie Narodowe's source commentary (p. I7) with the explanation provided here regarding Chopin's notational habits. 


\section{1 pitch notation}

a) stable disposition of pitches on staves (e.g. stem directions)

b) flexible placement in $\zeta$ or 9: and under ottava

\section{2 articulation}
a) frequent omission
b) relationship between centred/off-centre expression marks

\section{3 pedalling}
a) tendency to shift ${ }_{200}$ and $\oplus$ to right
b) tendency towards less pedalling (overall number/changes)
c) relationship between $\oplus_{\mathrm{S}}$ at end of $\mathrm{LH}$ figuration/end of bar

\section{4 slurring}
a) general stability across pairs of manuscripts (so $2+1$ or $1+2$ )
b) position of slurs (above/below/mixed) and beginning/end points
c) notational inaccuracies $\rightarrow$ new readings?

\section{5 dynamics}
a) tendency to lengthen hairpins (see 6a below)
b) relationship between $\mathrm{RH} / \mathrm{LH}$ dynamics; between cres, cres cres - - - -, and $\sim$ cres (likewise dim); between symbols centred/off-centre on figuration or in bar; between $>$ (long accent) and $<$
c) layout issues: cramped notation, oversize handwriting

\section{6 long accents}
a) tendency to extend long accents to 'diminuendos' (see 5a)
b) position above/below material and with regard to $\mathrm{RH} / \mathrm{LH} /$ both
c) treatment of conjunct long accents (e.g. $f>, f f>, f z>$ )

TABLE 8 Summary of notational characteristics revealed by comparing the Barcarolle sources 
in Table 8 pave the way for further investigation. Without new research in this vein, the notational lexicon and syntax to which I have referred will remain elusive, and current and future editorial initiatives and indeed our general understanding of the meaning and import of Chopin's music - including the smallest and seemingly most trivial strokes of his pen - will be correspondingly constrained. To fathom Chopin the composer as well as Chopin the copyist requires ongoing consideration of both the musical content and the notational detail of his music, with the proviso that often these turn out to be one and the same, or at least mutually indistinguishable. 


\section{Inhalt}

Vorwort 8

INTERPRETATION - BEGRIFF, METHODE, PRAXIS

Laure Spaltenstein Interpretation als treue Übersetzung. Zur Frühgeschichte eines vieldeutigen Begriffs I5

Kai Köpp Von der Quelle zur Methode. Zum Entwurf einer historischen Interpretationsforschung $\quad 28$

Manuel Bärtsch >Interpretation‘. Beethovens Sonate A-Dur op. IOI in der Sicht von Eugen d'Albert und Frederic Lamond

Sebastian Bausch Klavierrollen als Interpretationsdokumente. Ein Erfahrungsbericht als Leitfaden für Einsteiger $\quad 7 \mathrm{I}$

Camilla Köhnken Beethoven-Auslegung zwischen Liszts »Deklamationsstil« und Bülows »Vivisektionsversuchen«. Auf den Spuren Liszt'scher Interpretationsideale in Hans von Bülows instruktiver Edition der Klaviersonaten Beethovens $\quad 92$

Neal Peres Da Costa Carl Reinecke's Performance of his Arrangement of the Second Movement from Mozart's Piano Concerto K. 488. Some Thoughts on Style and the Hidden Messages in Musical Notation

II4

Carolina Estrada Bascuñana Enrique Granados's Performance Style.

Visualising the Audible Evidence I5O

Lukas Näf Tempogestaltung in Weberns Sinfonie op. 2I I80

INTERPRETATION - AUFFÜHRUNGSGESCHICHTE

Christoph Moor "Ein so erklärtes Lieblingsstück der hiesigen Kunstfreunde«. Die Rezeptionsgeschichte der Jupiter-Sinfonie in Beethovens Wien

Luisa Klaus Objektive Bruckner-Interpretation? Zur Aufführung der Trio-Entwürfe für die Neunte Sinfonie I940 205

Chris Walton Von innen und von außen. Beethovens Neunte Sinfonie und die $>$ Wagner'sche< Dirigiertradition $\quad 2 \mathrm{I} 8$

Lena-Lisa Wüstendörfer Streit um Fidelio. Gustav Mahler und Felix Weingartner im Disput um Werktreue $\quad 238$

INTERMEZZO

Robert Levin Turning Point to Musical Modernity. Beethoven as Executor of the Legacy of C. P. E. Bach. Concert Lecture 249

INTERPRETATION - INSTRUMENTE, ANALYSE, EDITION

Martin Skamletz «Man hat diese Erweiterung des Tonumfanges seit ein paar Jahren an den Tasteninstrumenten sehr weit getrieben.« Der Umgang mit Grenzen beim späten Mozart und beim frühen Beethoven $\quad 263$ 
Stephan Zirwes Analyse und Interpretation. Adolph Bernhard Marx' Beethoven-Analysen 29I

Michael Ladenburger Was können wir aus Originalhandschriften von Beethoven für eine angemessene Interpretation lernen? 30I

Federica Rovelli Die Skizzenbuch-Ausgaben und ihre mögliche digitale Zukunft 3 I7

Johannes Gebauer Interpretationspraktische Stemmatik. Philologische Methoden in der Interpretationsforschung am Beispiel annotierter Notenausgaben von Rodes 24 Capricen und Beethovens Violinkonzert

John Rink Chopin Copying Chopin 349

Tomasz Herbut Alexander Goldenweiser und Beethovens Sonate op. IIo- eine Spurensuche 366

INTERPRETATION - KREATIVE ANEIGNUNG

Thomas Gartmann Beethoven als sein eigener Interpret. Gedanken zur Bearbeitung der Klaviersonate op. I4/ז für Streichquartett

Ivo Haag Die Sinfonien von Johannes Brahms - (auch) Klaviermusik?

Michael Lehner Das Orchester auf dem Klavier. Welte-Klavierrollen von Gustav Mahler und Richard Strauss als interpretationsanalytische Quellen

Roger Allen “That Is What Music Really Is". Richard Wagner's Reception of Beethoven's Piano Sonata in A Major Op. IOI $43 \mathrm{I}$

Daniel Allenbach Eine heroische< Neunte? Dmitri Schostakowitschs Neunte Sinfonie im Vergleich mit Ludwig van Beethoenvs Sinfonien Nr.3 und 9 44I

Simeon Thompson Beethoven und der Zweite Weltkrieg in der künstlerischen Reflexion der Nachkriegszeit. Stanley Kubricks A Clockwork Orange und Rolf Liebermanns Leonore 40/45 456

Michelle Ziegler Rettungsversuch im Jubiläumsjahr. Mauricio Kagels Aufarbeitung der Beethoven-Rezeption in der Ludwig van-Werkgruppe (I970) 465

Leo Dick Über den späten Beethoven zur >Postidentität،. Die Suche nach liminalen Räumen im gegenwärtigen Musiktheater am Beispiel von Matthias Rebstocks Berliner Produktion Büro für postidentisches Leben $\quad 476$

Elizabeth Waterhouse Choreographic Re-mix. William Forsythe's Trio (I996) and Beethoven's String Quartet No. I5 in a Minor Op. I32 487

László Stachó "Gradus ad Parnassum".

The Purgatory of Instrumental Technique

Namen-, Werk- und Ortsregister 522

Die Autorinnen und Autoren der Beiträge 


\section{Rund um BeEthoven \\ Interpretationsforschung heute • \\ Herausgegeben von Thomas}

Gartmann und Daniel Allenbach 


\section{MUSIKFORSCHUNG DER Hochschule der KÜnste Bern Herausgegeben von Martin Skamletz und Thomas Gartmann Band 14}


0 Dieses Buch ist in gedruckter Form im Dezember 2019 in erster Auflage in der Edition Argus in Schliengen/Markgräflerland erschienen. Gestaltet und gesetzt wurde es im Verlag aus der Seria und der SeriaSans, die von Martin Majoor im Jahre 2000 gezeichnet wurden. Gedruckt wurde es auf Eos, einem holzfreien, säurefreien, chlorfreien und alterungsbeständigen Werkdruckpapier der Papierfabrik Salzer im niederösterreichischen Sankt Pölten. Das Vorsatzpapier Caribic cherry wurde von Igepa in Hamburg geliefert. Rives Tradition, ein Recyclingpapier mit leichter Filznarbung, das für den Bezug des Umschlags verwendet wurde, stellt die Papierfabrik Arjo Wiggins in Issy-les-Moulineaux bei Paris her. Das Kapitalband mit rot-schwarzer Raupe lieferte die Firma Dr. Günther Kast aus Sonthofen im Oberallgäu, die auf technische Gewebe und Spezialfasererzeugnisse spezialisiert ist. Gedruckt und gebunden wurde das Buch von der Firma Bookstation im bayerischen Anzing. Im Internet finden Sie Informationen über das gesamte Verlagsprogramm unter www.editionargus.de, zum Institut Interpretation der Hochschule der Künste Bern unter www.hkb.bfh.ch/interpretation und www.hkb-interpretation.ch. Die Deutsche Nationalbibliothek verzeichnet diese Publikation in der Deutschen Nationalbibliografie; detaillierte bibliografische Daten sind im Internet über www.dnb.de abrufbar. (c) der zeitgleich erschienenen digitalen Version: die Autorinnen und Autoren, 20I9. Dieses Werk ist lizenziert unter einer Creative Commons Namensnennung-Nicht kommerziell 4.0 International Lizenz (CC BY-NC 4.o). DoI: https://doi.org/I0.26045/kp64-6I78 ISBN 978-3-93I264-94-9 